\title{
Eisenmangelanämie in Schwangerschaft und Wochenbett
}

\author{
Dr. Pia Ruisinger, Gynäkologin; Prof. Dr. Franz Kainer, Gynäkologe, Nürnberg
}

\section{Der Eisenbedarf steigt während der Schwangerschaft physiologisch an und kann unter Umständen weder durch Diät noch aus den Eisenspeichern des Körpers gedeckt werden. Der daraus resultierende Eisenmangel ist die häufigste Ursache für eine Anämie in der Schwangerschaft und post partum. Diese ist mit einer erhöhten mater- nalen und fetalen Morbidität und Mortalität verbunden.}

\section{Häufigkeit und Ursache der Eisenmangelanämie}

Die Anämie (Blutarmut) ist ein häufiges Erscheinungsbild in der Schwangerschaft. Physiologisch kommt es zu einer Verminderung des Hämoglobins um 2-4 g/dl aufgrund einer Hämodilution (stärkere Zunahme des Plasmavolumens bis zur 24. SSW als ebenfalls gesteigerte Erythrozytenmasse). Darüber hinaus entsteht eine Anämie durch eine verminderte Hämoglobinsynthese (z.B. Eisenmangel, Fehl-/Mangelernährung, chronische/akute Entzündungen), einen vermehrten Abbau (Hämolyse z.B. durch: Präeklampsie/HELLP, Hämoglobinopathien wie Sichelzellanämie, Infektionen) oder einen verstärkten Verlust (Blutung z.B. durch Hypermenorrhoe präkonzeptionell, akute Blutung bei Plazenta prävia etc.).

Die häufigste Ursache einer Anämie in der Schwangerschaft ist ein Eisenmangel, der mit einer Prävalenz weltweit von 20-80\% bei Frauen im gebärfähigen Alter vorliegt [1]. In Mitteleuropa geht man bei graviden Frauen von einer Prävalenz von 10-15\% und in Entwicklungsländern von 50-75\% aus [2].

\section{Risikofaktoren für einen Eisenmangel sind: \\ - erhöhter Eisenbedarf (Schwanger- schaft, Wachstumsalter) (Tab. 1)}

- sozioökonomische Faktoren (niederiger Sozialstatus, Migrantinnen, junge Schwangere)

- Ernährungsgewohnheiten (Fehlernährung, Alkoholismus)

- chronische Blutverluste (Hypermenorrhoe, gastrointestinale Blutung)

- rasche Schwangerschaftsfolge

- Eisenmangelanämie in vorangehender Schwangerschaft

- chronische Darmerkrankungen/ Malabsorptionssyndrom [3]

Die Ursache einer Anämie ist - trotz der Dominanz des Eisenmangels - multifaktoriell bedingt. Das Symptom Blutarmut bedarf über die einfache Hämoglobinbestimmung hinaus einer differenzierten anamnestischen und labordiagnostischen Abklärung (s.u.).

\section{Basisinformation zum Eisenmetabolismus}

Der tägliche Eisenbedarf in der Schwangerschaft ist mit $30 \mathrm{mg}$ etwa doppelt so hoch wie der Eisenbedarf einer nicht schwangeren Frau zwischen 19 und 50 Jahren mit $15 \mathrm{mg}$. Bei einer Ernährung mit ausgewogener Mischkost werden täglich 15-20 mg Eisen aufgenommen, überwiegend in Form von Fleisch und Wurstwaren, jedoch werden davon nur $1 \mathrm{mg}$ bis maximal $3 \mathrm{mg}$ Eisen vom Darm resorbiert. Der Eisenbedarf in der Frühschwangerschaft liegt bei $4-6 \mathrm{mg} / \mathrm{d}$, in der Spätschwangerschaft bei 6-7 mg/d. Somit entsteht also ein Defizit von etwa $3 \mathrm{mg}$ Eisen pro Tag, was zu einer Entleerung der Eisenspeicher führt [5].

Nach der Resorption im Darm wird das Eisen für den Transport an das Protein Transferrin gebunden, und über den Transferrinrezeptor von unreifen Erythrozyten aufgenommen. Hier wird 75\% des Eisens zur Synthese des eisenhaltigen Proteinkomplexes Hämoglobin benötigt, welches den Sauerstoff an die Erythrozyten bindet. Da Eisen in freier Form die Zelle schädigt, liegt das restliche Eisen in seiner Speicherform Ferritin in Knochenmark, Leber und Milz vor oder dient der Enzymproduktion [6] (Abb. 1).

\section{Symptome und Risiken}

Eine Anämie wird meist aufgrund der Routinebestimmung des Hämoglobingehalts im Rahmen der Schwangerschaftsvorsorge diagnostiziert und ist zu dem Zeitpunkt häufig klinisch symptomlos.

Durch den erhöhten Eisenbedarf in der Schwangerschaft, der weder durch Diät noch aus den Eisenspeichern des Körpers gedeckt werden kann, kommt es u.a. zu 
Tab. 1 Negative Eisenbilanz in der Schwangerschaft [4].

\begin{tabular}{|l|r|}
\hline Fetus & $270 \mathrm{mg}$ \\
\hline Plazenta & $90 \mathrm{mg}$ \\
\hline Zunahme der Erythrozyten & $450 \mathrm{mg}$ \\
\hline Geburt (Blutung) & $150 \mathrm{mg}$ \\
\hline Nettoverlust von bis zu & $\mathbf{1 0 0 0} \mathbf{m g}$ \\
\hline
\end{tabular}

einer Beeinträchtigung der Synthese von Hämoglobin und somit zur Verminderung der Sauerstofftransportkapazität eines Erythrozyten. Da ein Erythrozyt eine Lebensdauer von etwa 120 Tagen hat, müssen folglich in Schwangerschaft und Wochenbett Erythrozyten neu gebildet werden, die jedoch durch den Substratmangel Eisen einen geringeren Hämoglobingehalt haben. Somit kommt es zur Minderversorgung der Peripherie mit Sauerstoff und kompensatorisch zur Hyperventilation und Hyperzirkulation.
Die sich daraus ergebenden Symptome sind allerdings meist unspezifisch und korrelieren nicht mit dem Schweregrad der Anämie.

\section{Klinische Symptome:}

- Reduzierte körperliche und geistige Leistungsfähigkeit

- Schwindel, Kopfschmerzen, Ohrensausen

- Belastungs- und Ruhedyspnoe

- Herzklopfen, Tachykardie, weite Blutdruckamplitude, funktionelle Herzgeräusche

- Restless-Legs-Syndrom

- blasse Haut und Schleimhaut [7]

Zusätzlich kommt es in Abhängigkeit vom Schweregrad der Anämie zu einer Zunahme von Schwangerschaftskomplikationen:

- Aborte

- Frühgeburten

- intrauterine Wachstumsretardierung
- intrauteriner Fruchttod

- maternale Harnwegsinfektionen [8]

Die Eisenmangelanämie korreliert außerdem bei Wöchnerinnen mit depressiven Verstimmungen und Stress. Diese Veränderungen können dazu führen, dass Mütter die Versorgung ihrer Säuglinge vernachlässigen und dadurch die Mutter-Kind-Beziehung gestört wird.

\section{Diagnostik}

Per definitionem ist eine Anämie eine Verminderung der Hämoglobin-Konzentration (Hb) im Blut unter die alters- und geschlechtsspezifische Norm. Für die Schwangerschaft ergeben sich nach WHO und CDC (Center of Disease Control) folgende Grenzwerte:

- Gynäkologie: $\mathrm{Hb}<12$ g/dl

- Schwangerschaft I./III. Trimenon: $\mathrm{Hb}<11 \mathrm{~g} / \mathrm{dl}$ 


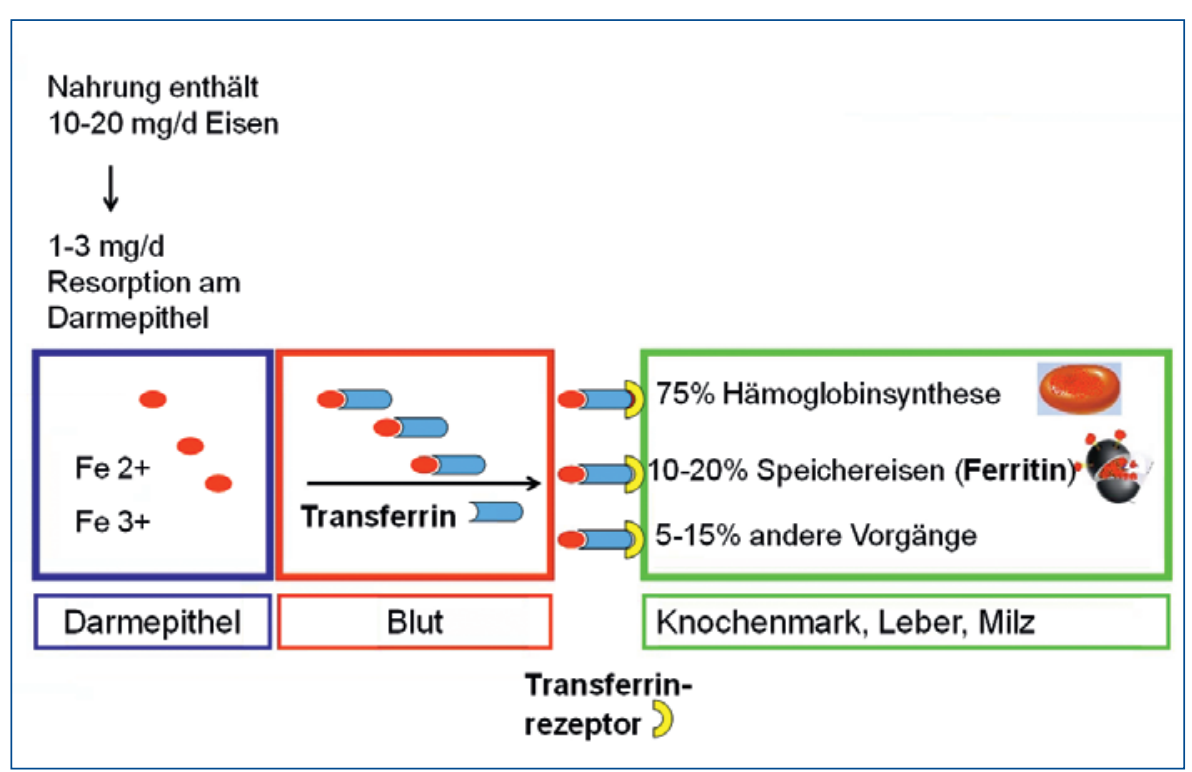

Abb. 1 Eisenstoffwechsel (eigene Darstellung).

$$
\begin{aligned}
& \text { - Schwangerschaft II. Trimenon: } \\
& \mathrm{Hb}<10,5 \mathrm{~g} / \mathrm{dl} \\
& \text { - Post partum: } \mathrm{Hb}<10 \mathrm{~g} / \mathrm{dl}[9]
\end{aligned}
$$

Bislang beschränkte sich die Diagnostik weitgehend auf die Bestimmung des Hämoglobinwerts im Blut. Dieser Wert kann jedoch keine Auskunft über den aktuellen Eisenstatus geben. Da ein Fließgleichgewicht zwischen dem Ferritin in den Zellen und im Serum besteht, erlaubt die Ferritinbestimmung im Serum eine Aussage über den Eisenbestand des Körpers.

Vier Befundgruppen sind bei der Ferritinbestimmung relevant [10]:

$-<15 \mu \mathrm{g} / \mathrm{l}$ : Eisenspeicher entleert

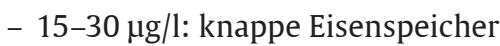

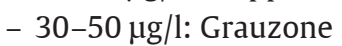

- > $50 \mu \mathrm{g} / \mathrm{l}$ : genügend Eisenspeicher

Bei Leberschäden, Tumorerkrankungen und aktiven Entzündungen kann der Wert jedoch auch unabhängig vom Speichereisen erhöht sein, da Serumferritin ein Akutphaseprotein ist.

Hier können der lösliche Transferrinrezeptor oder Erythrozytenindizes wie MCV (mittleres Zellvolumen eines Erythrozyten), MCHC (mittlere Hämoglobinkonzentration eines Erythrozyten) sowie
Retikulozytenzählung Aufschluss über einen Eisenmangel geben. Außerdem empfiehlt sich eine CRP-Bestimmung, um einen Eisenmangel bei entzündungsbedingt hohen Serumferritin-Werten ggf. zu demaskieren.

\section{Prophylaxe und Therapie}

Generell richtet sich die Therapie der Anämie nach deren Ursache und Schwere. Für die Auswahl der Therapieoption muss die tolerierbare Zeitspanne für einen positiven Therapieeffekt $v$.a. kurz vor Entbindung und bei schweren postpartalen Blutungen berücksichtigt werden.

Im Allgemeinen ist eine Anämie in der Schwangerschaft behandlungsbedürftig, wenn das Hämoglobin einen Wert von $<10,5 \mathrm{~g} / \mathrm{dl}$ und das Serumferritin einen Wert von $<30 \mu \mathrm{g} / \mathrm{l}$ unterschreitet [10].

Durch eine Diät mit eisenangereicherten Nahrungsmitteln kann das Risiko für die Entstehung eines Eisenmangels vermindert werden. Zur Therapie eines bestehenden Eisenmangels sind diese jedoch nicht ausreichend. Grund dafür ist die eingeschränkte Bioverfügbarkeit von
Eisen, welche von vier Faktoren bestimmt wird: die Wertigkeit des Eisenions (zweiwertig oder dreiwertig), die Herkunft (tierisch, pflanzlich, mineralisch), die Löslichkeit und die Art der Bindung im Molekül (gebunden an Proteine, Gerbstoffe). In pflanzlichen Lebensmitteln liegt dreiwertiges Eisen vor und ist teilweise fest an bestimmte Kohlenhydrate gebunden, sodass es generell schlecht bioverfügbar ist. Es wären große Mengen nötig, um den täglichen Bedarf zu decken. In Fleisch, Fisch und Geflügel liegt zweiwertiges Eisen vorwiegend gebunden an Myo- und Hämoglobin (sogenanntes Hämeisen) vor, das durch einen speziellen Rezeptor an der Darmzelle vergleichsweise gut resorbiert werden kann [6] (Tab. 2).

Eine allgemeingültige Richtlinie der Fachgesellschaften zur Eisensubstitution in der Schwangerschaft existiert derzeit in Deutschland noch nicht. In der gängigen Literatur sind sich die Autoren jedoch einig, dass der erhöhte physiologische Eisenbedarf vor allem in der 2. Hälfte der Schwangerschaft nicht allein über die Nahrung gedeckt werden kann. Eine Eisensubstitution während der Schwangerschaft führt zu einer Erhöhung der Ferritin- und HämoglobinKonzentration und somit zu einer Verminderung der Prävalenz der Eisenmangelanämie [10].

Ist die Diagnose einer Eisenmangelanämie in der Schwangerschaft anhand der Parameter Hämoglobin und Serumferritin gestellt, sollte anhand eines Stufenschemas die richtige Therapie gewählt werden.

Zur Auswahl stehen: orale Eisensubstitution, parenterale Eisengabe, Bluttransfusion (Tab.3).

\section{Orale Eisensubstitution}

Bei bestehender Indikation ist die Verabreichung von Eisen-II-Sulfat 1-2× $\mathbf{8 0 ~ m g / d ~ o r a l ~ o d e r ~ m i t ~ A ̈ q u i v a l e n t p r a ̈ p a - ~}$ rat für die Dauer von 6 Wochen etwa 1-2 
Tab. 2 Bioverfügbarkeit von Eisen in Lebensmitteln.

\begin{tabular}{|l|l|l|l|}
\hline Nahrungsmittel & Zubereitung & Eisengehalt (mg/100g) & Bioverf \\
\hline Tierische Nahrungsmittel, Hämeisen & & & \\
\hline - Rindfleisch & roh, frisch & 2,0 bis 4,3 & hoch \\
\hline - Schweinefleisch & roh, frisch & 2,5 bis 5,0 & hoch \\
\hline - Geflügel & roh, frisch & 1,5 bis 2,5 & hoch \\
\hline - Fisch & roh & 0,5 bis 1,0 & hoch \\
\hline Tierische Nahrungsmittel, Nicht-Hämeisen & & & niedrig \\
\hline - Leber & roh & 6,0 bis 14,0 & niedrig \\
\hline - Ei & ganz, frisch & 2,0 bis 3,0 & mittel \\
\hline - Kuhmilch & Frisch, Vollmilch & 0,1 bis 0,4 & \\
\hline Pflanzliche Nahrungsmittel & & & niedrig \\
\hline - Hülsenfrüchte & variabel & 1,9 bis 14,0 & niedrig \\
\hline - Getreidemehl & Vollkorn & 4,0 & niedrig \\
\hline - Getreidemehl & Weißmehl & 1,5 & niedrig \\
\hline - Getrocknete Früchte & variabel & 2,0 bis 10,6 & niedrig \\
\hline - grünes Blattgemüse & roh & 0,4 bis 18,0 & niedrig \\
\hline - Wurzelpflanzen & roh & 0,3 bis 2,0 & niedrig \\
\hline - Früchte & Konserven oder frisch & 0,2 bis 4,0 &
\end{tabular}

Stunden vor den Mahlzeiten Mittel der ersten Wahl [8]. Zusätzlich wird bei Verträglichkeit die Einnahme von Vitamin C empfohlen, um die enterale Eisenresorption zu optimieren. Eine weitere Dosissteigerung ist nicht zu empfehlen, da sie nicht zu einer höheren enteralen Resorption, jedoch zu häufigeren Nebenwirkungen führt. Diese sind v.a. gastrointestinale Symptome wie Oberbauchschmerzen, Übelkeit, Sodbrennen, Obstipation, dunkler Stuhl, aber auch Durchfall und unspezifische Unverträglichkeitsreaktionen. Der Therapieerfolg kann anhand eines Retikulozytenanstiegs ab dem 4. Therapietag, einem Hämoglobinanstieg nach 10-14 Tagen oder dem Anstieg des Ferritinspiegels auf 50-80 $\mu \mathrm{g} / \mathrm{l}$ bewertet werden [1]].

\section{Parenterale Eisensubstitution}

Bei schlechter Verträglichkeit oder Erfolglosigkeit der oralen Therapie sowie bei schwerer Eisenmangelanämie ( $\mathrm{Hb}$ $<9 \mathrm{~g} / \mathrm{dl}$, Serumferritin $<12 \mu \mathrm{g} / \mathrm{l}$ ) ist die intravenöse Eisengabe aufgrund ihrer deutlich höheren Effektivität zu bevorzugen. Die Dosierung muss individuell dem Gesamteisendefizit angepasst werden und errechnet sich nach der GanzoniFormel:

Eisendefizit $(\mathrm{mg})=$ Körpergewicht $(\mathrm{kg}) \times$ (Soll-Hb g/dl - Ist-Hb g/dl) × 2,4 + Reserveeisen (500 $\mathrm{mg}$ )
So müssten zum Beispiel bei einer Patientin mit $70 \mathrm{~kg}$ Körpergewicht ca. $700 \mathrm{mg}$ Eisen appliziert werden, um einen $\mathrm{Hb}$-Anstieg von $1 \mathrm{~g} / \mathrm{dl}$ zu erreichen.

Zur parenteralen Eisentherapie steht eine Reihe von intravenösen Präparaten zur Verfügung. Aufgrund des besten Nebenwirkungs-Wirkungs-Profils wird eine Therapie mit Fe(II)-hydroxid-Polymaltose $100-1000 \mathrm{mg}$ in $50-100 \mathrm{ml} \mathrm{NaCl}$ $0,9 \%$ als einmalige Kurzinfusion (max. Wochendosis $1000 \mathrm{mg}$ ) oder Eisensac-

Tab. 3 Stufenschema einer Eisenmangelanämie in der Schwangerschaft $[10,11]$.

\begin{tabular}{|c|c|}
\hline Schwere der Anämie & Therapie \\
\hline $\begin{array}{l}\mathrm{Hb}<10,5 \mathrm{~g} / \mathrm{dl} \text { und } \\
\text { Serumferritin }<30 \mu \mathrm{g} / \mathrm{l}\end{array}$ & Orale Eisengabe: Eisen-II-Sulfat (Tardyferon ${ }^{\circledR}$ ) $160 \mathrm{mg} / \mathrm{d}$ \\
\hline $\begin{array}{l}\mathrm{Hb}<9 \mathrm{~g} / \mathrm{dl} \text { und } \\
\text { Serumferritin }<12 \mu \mathrm{g} / \mathrm{l}\end{array}$ & $\begin{array}{l}\text { Parenterale Eisengabe: Eisen-Polymaltose }\left(\text { Ferinject }{ }^{\circledR}\right) \\
\text { einmalig bis } 1000 \mathrm{mg} \text { i.v. } 1 \times / \text { Woche oder Eisensaccharose } \\
\left(\text { Venofer }^{\circledR}\right) 200 \mathrm{mg} \text { i.v. } 1-2 \times / \text { Woche }\end{array}$ \\
\hline $\mathrm{Hb}<7 \mathrm{~g} / \mathrm{dl}$ & Bluttransfusion \\
\hline
\end{tabular}


charose $200 \mathrm{mg}$ in $200 \mathrm{ml} \mathrm{NaCl} \mathrm{0,9 \%} \mathrm{als}$ Kurzinfusion 1-2×/Woche (max. Wochendosis $1600 \mathrm{mg}$ ) empfohlen. Als Nebenwirkung kann es zu einer FlushReaktion und generalisierten Hauteffloreszenzen, zu Kopfschmerzen oder zu einem metallischen Mundgeschmack während der Kurzinfusion kommen [10].

\section{Bluttransfusion}

Eine Transfusion von Erythrozytenkonzentraten darf nur nach ausführlicher Aufklärung der Schwangeren über Notwendigkeit und Risiken der Transfusion und nach strenger Indikationsstellung durchgeführt werden. Eine Fremdblutgabe sollte möglichst unter Berücksichtigung der Patientenwünsche erfolgen, allerdings muss bei vitaler Indikation der Ernst der Situation unmissverständlich kommuniziert werden.

Die Bluttransfusion ist die sicherste Methode, eine Anämie zeitnah zu therapieren. Weist eine schwangere Patientin mit Anämie akute Blutvolumenmangelsymptome auf, besteht die Indikation zur Fremdbluttransfusion. Ebenso sollte bei hämodynamisch stabilen Schwangeren eine Fremdbluttransfusion spätestens ab einem $\mathrm{Hb}<7 \mathrm{~g} / \mathrm{dl}$ angeboten werden, bzw. ab einem $\mathrm{Hb}<9 \mathrm{~g} / \mathrm{dl}$, falls geburtshilfliche Blutungsrisiken vorhanden sind [11].

\section{Anämie im Wochenbett}

Eine therapiepflichtige Anämie im Wochenbett liegt bei Frauen mit einem $\mathrm{Hb}<10 \mathrm{~g} / \mathrm{dl}$ vor. Die Bestimmung des Hb-Wertes sollte 24-48 Stunden postpartum bei Blutverlusten $>500 \mathrm{ml}$ peripartal erfolgen [11]. In der Regel handelt es sich hierbei um eine akute Blutungsanämie, welche durch einen vorbestehenden Eisenmangel weiter verstärkt wurde. Symptome der postpartalen Anämie können eine erhöhte kardiovaskuläre Belastung (orthostatische Beschwerden, Schwindel), eine verminderte Leistungsfähigkeit und schnelle
Ermüdung, sowie eine verzögerte Wundheilung und erhöhtes Infektionsrisiko sein.

Auch hier stehen die drei Varianten der Therapie - oral, parenteral, Transfusion - zur Verfügung (Tab.4).

Eine orale Eisensubstitution ist oft nicht ausreichend, da die Eisenspeicher weitgehend entleert sind und gleichzeitig die Eisenresorption durch postpartale Entzündungsreaktionen vermindert wird. In diesen Fällen wird die parenterale Applikation von Eisen empfohlen. Die Gabe von Erythrozytenkonzentraten muss streng indiziert sein und sollte nur im Notfall oder bei schweren, klinisch symptomatischen Anämien erfolgen. Unabhängig vom Hämoglobinwert muss die Infusion von Plasmaexpandern und kristalloiden Lösungen zur Kreislaufstabilisierung großzügig appliziert werden.
Eine Anämiebehandlung mit Erythropoetin sollte nur im Rahmen wissenschaftlicher Studien durchgeführt werden [11].

\section{Schwangerschaftsvorsorge}

Im Rahmen der Mutterschaftsrichtlinien wird die Hb-Konzentration in der Schwangerschaft regelmäßig bestimmt und kann somit Aussage über das Vorliegen einer Anämie geben. Sie sagt jedoch nichts über die Kapazität der körpereigenen Eisenspeicher aus. Eine Ferritinbestimmung kann Aufschluss über eine ausreichende Füllung der Eisenspeicher geben, noch bevor sich eine Anämie manifestiert (Normbereich $50-80 \mu \mathrm{g} / \mathrm{l})$.

Die Prophylaxe eines Eisenmangels und einer daraus resultierenden Eisenmangelanämie ist sinnvoll und effektiv [13]. 
Tab. 4 Stufenschema einer Anämie im Wochenbett [10].

\begin{tabular}{|c|c|}
\hline Schwere der Anämie & Therapie \\
\hline $\mathrm{Hb}<10 \mathrm{~g} / \mathrm{dl}$ & $\begin{array}{l}\text { Orale Eisengabe: Eisen-II-Sulfat (Tardyferon }{ }^{\circledR} \text { ) } \\
200 \mathrm{mg} / \mathrm{d}\end{array}$ \\
\hline $\begin{array}{l}\mathrm{Hb}<8 \mathrm{~g} / \mathrm{dl} \text { oder } \\
\text { Serumferritin }<12 \mu \mathrm{g} / \mathrm{l}\end{array}$ & $\begin{array}{l}\text { Parenterale Eisengabe: Eisen-Polymaltose } \\
\left.\text { (Ferinject }^{\circledR}\right) 1000 \mathrm{mg} \text { einmalig i.v. oder } \\
\text { Eisensaccharose }\left(\text { Venofer }^{\circledR}\right) 200 \text { mg i.v. über } \\
\text { 2-4 Tage }\end{array}$ \\
\hline $\begin{array}{l}\text { Schwere peripartale Blutverluste mit } \\
\text { Symptomen des Volumenmangels }\end{array}$ & Bluttransfusion \\
\hline
\end{tabular}

\section{Aufgrund der fehlenden Richtlinien der Fachgesellschaften zur Therapie und Prophylaxe der Eisenmangelanämie in Schwangerschaft und Wochenbett gibt es allerdings keine eindeutige Empfeh- lung zur Eisensubstitution.}

Aus unserer Sicht ist eine generelle Eisenmangelprophylaxe mit 30-40 mg Eisen pro Tag bei allen Schwangeren ab der 10. SSW einer ferritinspiegelabhängigen Eisensubstitution vorzuziehen [11]. Von wesentlicher Bedeutung ist eine ausführliche Beratung der Patientin präkonzeptionell, in der Schwangerschaft, sowie im Wochenbett. Diese sollte individuell und abhängig von der jeweiligen medizinischen und sozialen Situation erfolgen.

\section{Literatur}

1 Breymann C et al. Semin Hematol 2015 Oct; 52 (4): 339-347. doi: 10.1053/j. seminhematol.2015.07.003. Epub 2015 Jul 10. Iron Deficiency Anemia in Pregnancy
2 Shrimpton R et al. Food Nutr Bull 2009 Dec; 30 (4 Suppl): 556-573. Multiple micronutrient supplementation during pregnancy in developing-country settings: policy and program implications of the results of a meta-analysis

3 Breymann C et al. Eur J Intern Med 2012 Sep; 23 (6): 529-533. doi: 10.1016/j. ejim.2012.04.008. Epub 2012 May 4. Anemia-prevalence and risk factors in pregnancy

4 Bothwell. Am J Clin Nurt 2000; 72 (suppl): 257S-264S

5 Scholl TO. Iron status during pregnancy: setting the stage for mother and infant. Am J Clin Nutr 2005; 81: 1218S-1222S

6 Bastigkeit M. Eisen - Neue Erkenntnisse verändern Therapie, GOVI-Verlag 2005

7 Schleußner E. Eisenmangel und Eisenmangelanämie in der gynäkologischen Praxis. Therapiecard Eisenmangelanämie. Stuttgart: Georg Thieme Verlag: 2013

8 Schaefer RM et al. Aktuelle Empfehlungen zur Therapie der Eisenmangelanämie - update 2005. Eisenbrief 2005; 3: 1-12. Verfügbar auf www.journalmed.de
9 Breymann C. Milk iron content in breast-feeding mothers after administration of intravenous iron sucrose complex. Perinat Med 2007; 35 (2): 115-118

10 Bergmann RL et al. Diagnostik und Behandlung der Anämie und des Eisenmangels in der Schwangerschaft und im Wochenbett. Geburtsh Frauenheilk 2009; 69: 682-686

11 Dudenhausen J. Eisenmangel rasch identifizieren. Dtsch Aerztebl 2010; 107 (25): A-1270

12 Milman N, Hertz J. Pregnancy and iron prophylaxis - how an how much? Ugeskr Laeger 2010; 172: 433-436

13 Peña-Rosas JP et al. Intermittent oral iron supplementation during pregnancy. Cochrane Database Syst Rev 2015

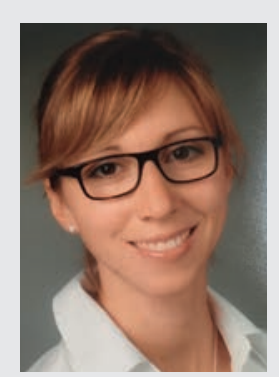

Dr. med. Pia Ruisinger Geburtshilfe \& Gynäkologie Klinik Hallerwiese St.-Johannis-Mühlgasse 19 90419 Nürnberg E-Mail: pia.ruisinger@ diakonieneuendettelsau.de 